\title{
Resultados de sedentarismo en la población de primer semestre de 2010 de la Universidad Santo Tomás ${ }^{1}$
}

\author{
Ana María Torres Pazmiño*, Henry Humberto León Ariza*, Ianellen Arias Padilla***
}

\begin{abstract}
Recibido: 12 de mayo de 2010 Revisado: 25 de junio de 2010 Aprobado: 10 de agosto de 2010
\end{abstract}

\begin{abstract}
RESUMEN
Los resultados de múltiples investigaciones han permitido determinar que las personas físicamente activas son mucho más saludables que los sedentarios. Se evaluaron 1.140 estudiantes de primer semestre a través de una prueba estandarizada propuesta por Bouchard en 1983; posteriormente se calculó de forma indirecta el gasto energético diario y se determinó el número de estudiantes que invierten menos del $10 \%$ de su gasto energético en actividades físicas de 4 o menos unidades de índice metabólico (MET). El porcentaje de sedentarismo de los estudiantes evaluados fue del $68.7 \%$, (77.4\% mujeres y $60.1 \%$ hombres); el programa con mayor grado de sedentarismo fue Ingeniería de Telecomunicaciones y la menor fue Cultura Física. Organizando los datos por grupos etarios, se encontró que el 91.8\% de los estudiantes se encuentra entre los 16 y 21 años y que en todos los grupos es mayor el sedentarismo en las mujeres que en los hombres. Comparado con otros estudios, los resultados de sedentarismo son ligeramente menores, pero no dejan de ser preocupantes. Si no se adoptan rápidamente medidas que combatan su origen y eviten su desarrollo, la situación en términos de salud pública va a ser muy difícil en los próximos años.
\end{abstract}

\section{Palabras claves}

Composición corporal, sedentarismo, estudiantes universitarios.

1 Artículo de investigación realizado por el área de salud de la Cátedra de Cultura Física (Gruplac), actual Cátedra de Formación Física Integral Henri Didón, del Departamento de Humanidades y Formación Integral de la Universidad Santo Tomás, sede Bogotá. Línea de Investigación: Cátedra institucional Henry Didón. El estudio presentado aquí fue realizado con recursos del Fondo para el Desarrollo de la Investigación de la Universidad Santo Tomás (FODEIN-USTA).

* Especialista en Administración Deportiva, Profesional de la Cultura Física, Deporte y Recreación de la Universidad Santo Tomás, sede Bogotá. Docente de la Cátedra de Formación Física Integral Henri Didón del Departamento de Humanidades y Formación Integral de la Universidad Santo Tomás. Forma parte del grupo de investigación del área de la salud Cátedra de Cultura Física. Correo electrónico: paezani@ yahoo.com

** Licenciado en Educación física de la Universidad Pedagógica Nacional. Médico Cirujano de la Universidad Nacional de Colombia. Coordinador del área de la salud: Cátedra de Formación Física Integral Henri Didón y docente director de la línea de investigación en fisiología muscular: Facultad de Cultura Física, Deporte y Recreación, Bogotá. Correo electrónico: preparacionfisica2@ gmail.com

*** Licenciada en Educación Física de la Universidad del Quindío. Fisioterapeuta de la Corporación Universitaria Iberoamericana de Bogotá. Docente de la Cátedra de Formación Física Integral Henri Didón, del Departamento de Humanidades y Formación Integral de la Universidad Santo Tomás. Forma parte del grupo de investigación del área de la salud Cátedra de Cultura Física. Correo electrónico: iapa81@ hotmail.com 


\title{
Results of the physical inactivity in freshmen of the first semester 2010 at the Universidad Santo Tomás
}

\author{
Ana María Torres Pazmiño, Henry Humberto León Ariza, Ianellen Arias Padilla
}

\begin{abstract}
The results of numerous investigations have established that physically active people are much healthier than sedentary people. 1140 freshmen were assessed through a standardized test proposed by Bouchard in 1983; then, the daily energy was indirectly calculated and the number of students who spend less than $10 \%$ of energy expenditure in physical activites of 4 or less units of metabolic rate (MET) was determined. The percentage of sedentary students tested was $68.7 \%$ (77.4\% women and $60.1 \%$ men); the program with the highest degree of sedentarism was Telecommunications Engineering and Physical Culture had the least sedentarism. By organizing the data by age groups, it was possible to see that $91.8 \%$ of students lie between 16 and 21 years and that the percentage of sedentarism in all groups is greater in women than in men. Compared to other studies of physical inactivity, the results are slightly lower, but they are still disturbing. If actions are not taken to struggle against its origin and prevent its development, the situation in terms of public health will be very difficult in coming years.
\end{abstract}

\section{Key words}

Body composition, sedentarism, college students.
Recibido: 12 de mayo de 2010 Revisado: 25 de junio de 2010 Aprobado: 10 de agosto de 2010 


\section{INTRODUCCIÓN}

La evidencia científica existente, referente a los efectos deletéreos del sedentarismo, es cada día mayor, y el resultado de múltiples investigaciones ha permitido reconocer los mecanismos fisiológicos desencadenados por la actividad física que participan en la prevención y tratamiento de múltiples enfermedades y, por ende, en una mejora de la calidad de vida. Algunos datos indican que tanto hombres como mujeres que son físicamente activos reducen su riesgo de morir por enfermedad cardiovascular entre un $20 \%$ a un $35 \%$ (Darren, Warburton \& Shannon, 2006), así como la probabilidad de sufrir de cáncer de colon entre un 30 a 40\% y de seno en un 30\% (Darren et al., 2006), disminuyen las complicaciones de la diabetes mellitus tipo 2 (especialmente las cardiovasculares) entre un 30 a 50\% (Bassuk \& Joann, 2005); controlan los valores de presión arterial, el peso corporal y la cantidad de grasa visceral (Mathieu, Poirier, Pibarot, Lemieux \& Després, 2009); reducen la cantidad de colesterol de baja densidad (el perjudicial) y los triglicéridos, al tiempo que aumentan el colesterol de alta densidad (el beneficioso; Thompson, Buchner \& Piña, 2003), entre otras.

A pesar de lo benéfico del ejercicio físico, los datos actuales muestran que la prevalencia de sedentarismo es muy alta. En Colombia, el estudio más grande lo realizó el Instituto Colombiano de Bienestar Familiar ([ICBF], 2006) ${ }^{1}$. Según esta encuesta, sólo el $26 \%$ de la población $(27.6 \%$ hombres y $24.2 \%$ de las mujeres) entre 13 y 17 años cumple con los requerimientos establecidos de actividad fí-

1 El estudio se denominó: "Encuesta nacional de la situación nutricional en Colombia" (ENSIN) y fue realizada y publicada en el año 2005 . sica para su edad. En Bogotá, sólo el 25.1\% de individuos entre 18 a 64 años se considera activo físicamente (ICBF, 2006).

A través de este estudio se pretende presentar datos actualizados sobre sedentarismo en población universitaria de primer semestre; posterior a ello, se realizará un análisis de los resultados con una propuesta de mejoramiento y promoción de la actividad física.

\section{Metodología}

Entre los 1.510 estudiantes matriculados en el primer semestre del año 2010 en la Universidad Santo Tomás, se realizó en 1.140 estudiantes una prueba estandarizada para determinar el grado de sedentarismo por medio del método de diario. Para este fin, se utilizó la encuesta de MET propuesta por Bouchard, Tremblay, Leblanc, Lortie, Savard \& Thériault (1983). La cuantificación de gasto energético en grupos de adultos presenta un error no mayor al 6\%, generalmente 3\%, con tendencia a sobreestimar levemente el gasto energético; el error individual calculado está entre el 7\% y el 8\% (Montoye, Kemper, Saris \& Washburn, 1996).

\section{Instrumento}

Para la evaluación se aplicó una encuesta que evaluaba el gasto calórico aproximado durante 24 horas cada 15 minutos. Para esto, el evaluado debía escoger dos días entre semana y uno de fin de semana para responder con números de 1 a 9 cada una de las casillas. Cada uno de los números correspondía a las actividades realizadas, o su equivalente: 1 (dormir, descansar acostado) hasta 9 (trabajos manuales de alta intensidad, carreras a una velocidad superior a los $9 \mathrm{~km} / \mathrm{h}$, 
entre otras). La aplicación de la encuesta incluyó la aplicación de un cuestionario, que fue contestado previamente por un grupo piloto de docentes de la Cátedra de Formación Física Integral Henri Didón, lo que permitió su revisión y corrección, además este sirvió para que todos los docentes conocieran la metodología.

\section{Interpretación}

Con la encuesta completamente diligenciada, se procedió a contar el número total de cada uno de los valores (cantidad de uno, cantidad de dos, etc.). Este número se podía convertir a MET o a Kcal/día. Si una persona invierte menos del 10\% de su gasto energético diario a la realización de actividades físicas que requieran al menos 4 MET o si dichas actividades no superan los 30 minutos se consideraba sedentario (Cabrera, Rodríguez, Rodríguez, Anía, Brito, Muros et al., 2007), de lo contrario se consideraba al estudiante como activo.

El análisis de los resultados se hizo mediante Excel 2007 (Microsoft Corporation) y el programa estadístico SPSS versión 17 (SPSS Inc.).
Desde el punto de vista ético, los métodos empleados son inocuos, por lo que se clasifica como una investigación con riesgo mínimo, según la Resolución 8430 de 1993 del Ministerio de Salud de la República de Colombia. Todo lo consignado fue estrictamente privado y confidencial.

\section{Resultados}

Las características generales de los estudiantes a quienes se les aplicó la encuesta se encuentran en la Tabla 1, lo cual demuestra que fue estudiado un número bastante similar de hombres y mujeres, que el rango de edad en el que se encuentra el mayor número de estudiantes es entre los 16 y 18 años con el $71.4 \%$, que el programa que más llenó encuestas fue el de Negocios Internacionales y el que menos lo hizo fue Ingeniería de Telecomunicaciones, y que un porcentaje bajo de estudiantes no consignó la edad $(0.5 \%)$ y otro tanto no colocó los datos de programa académico al que pertenecían (2.1\%). 
Tabla 1. Distribución por género, por edad y por facultad de los estudiantes que completaron la encuesta de MET.

\begin{tabular}{|c|c|c|}
\hline VARIABLE & $\mathbf{n}$ & $\%$ \\
\hline \multicolumn{3}{|l|}{ GÉNERO } \\
\hline HOMBRES & 577 & $50.6 \%$ \\
\hline MUJERES & 563 & $49.4 \%$ \\
\hline TOTAL & 1140 & $100.0 \%$ \\
\hline \multicolumn{3}{|c|}{ EDAD (AÑOS) } \\
\hline$<16$ & 8 & $0.7 \%$ \\
\hline $16-18$ & 814 & $71.4 \%$ \\
\hline $19-21$ & 232 & $20.4 \%$ \\
\hline $22-24$ & 54 & $4.7 \%$ \\
\hline$>25$ & 26 & $2.3 \%$ \\
\hline No informado & 6 & $0.5 \%$ \\
\hline TOTAL & 1140 & $100.0 \%$ \\
\hline \multicolumn{3}{|l|}{ FACULTAD } \\
\hline Ingeniería Ambiental & 91 & $8.0 \%$ \\
\hline Economía & 71 & $6.2 \%$ \\
\hline Comunicación Social & 110 & $9.6 \%$ \\
\hline Ingeniería Civil & 62 & $5.4 \%$ \\
\hline Negocios Internacionales & 177 & $15.5 \%$ \\
\hline Contaduría Pública & 67 & $5.9 \%$ \\
\hline Cultura física & 110 & $9.6 \%$ \\
\hline Ingeniería Electrónica & 31 & $2.7 \%$ \\
\hline Psicología & 58 & $5.1 \%$ \\
\hline Licenciatura en Filosofía y Lengua Castellana & 19 & $1.7 \%$ \\
\hline Derecho & 127 & $11.1 \%$ \\
\hline Ingeniería de Telecomunicaciones & 13 & $1.1 \%$ \\
\hline Administración de Empresas & 90 & $7.9 \%$ \\
\hline Sociología & 31 & $2.7 \%$ \\
\hline Ingeniería Mecánica & 41 & $3.6 \%$ \\
\hline Estadística & 18 & $1.6 \%$ \\
\hline No informado & 24 & $2.1 \%$ \\
\hline TOTAL & 1140 & $100.0 \%$ \\
\hline
\end{tabular}

El porcentaje de sedentarismo encontrado en los estudiantes analizados fue del $68.7 \%$, siendo Ingeniería de Telecomunicaciones la Facultad con mayor sedentarismo (92.3\%) y Cultura Física la que menos con $43.6 \%$.
Los resultados generales de sedentarismo por Facultades se encuentran en la Tabla 2 y su representación gráfica corresponde a la figura 1. 
Tabla 2. Distribución de estudiantes sedentarios por cada una de las facultades.

\begin{tabular}{|c|c|c|c|}
\hline FACULTAD & $\begin{array}{c}\text { TOTAL DE } \\
\text { ESTUDIANTES }\end{array}$ & $\begin{array}{c}\text { ESTUDIANTES } \\
\text { SEDENTARIOS }\end{array}$ & $\%$ SEDENTARISMO \\
\hline Ingeniería Ambiental & 91 & 56 & $61,5 \%$ \\
\hline Economía & 71 & 51 & $71,8 \%$ \\
\hline Comunicación Social & 110 & 68 & $61,8 \%$ \\
\hline Ingeniería Civil & 62 & 48 & $77,4 \%$ \\
\hline Negocios Internacionales & 177 & 131 & $74,0 \%$ \\
\hline Contaduría Pública & 67 & 53 & $79,1 \%$ \\
\hline Cultura física & 110 & 48 & $54,6 \%$ \\
\hline Ingeniería Electrónica & 31 & 17 & $79,3 \%$ \\
\hline Psicología & 58 & 46 & $78,9 \%$ \\
\hline $\begin{array}{c}\text { Licenciatura en Filosofía y } \\
\text { Lengua Castellana }\end{array}$ & 19 & 15 & $73,2 \%$ \\
\hline Derecho & 127 & 93 & $92,3 \%$ \\
\hline Ingeniería de & 13 & 12 & $73,3 \%$ \\
\hline Telecomunicaciones & 90 & 66 & $67,7 \%$ \\
\hline Adminstración de Empresas & 31 & 21 & $63,4 \%$ \\
\hline Sociología & 41 & 26 & $72,2 \%$ \\
\hline Ingeniería Mecánica & 18 & 13 & $79,2 \%$ \\
\hline Estadística & 24 & 19 & $\mathbf{6 8 , 7} \%$ \\
\hline No informado & $\mathbf{1 1 4 0}$ & 783 & \\
\hline TOTAL & & & \\
\hline
\end{tabular}

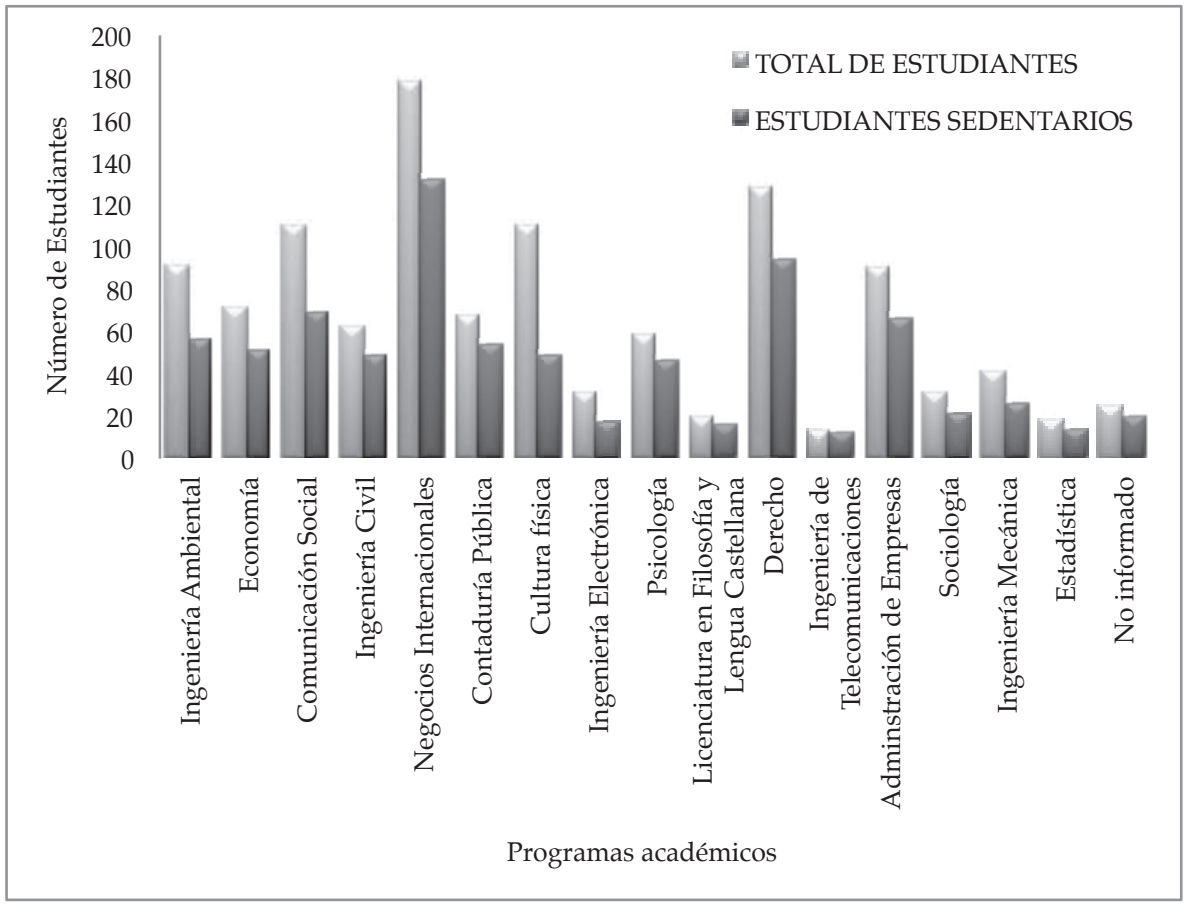

Figura 1. Representación gráfica de la distribución de estudiantes por cada una de las facultades. 
La distribución por género de los resultados demostró que el $77.4 \%$ de las mujeres son sedentarias contra un $60.1 \%$ de los hombres. En 11 de los 16 programas el porcentaje de mujeres sedentarias supera al de los hombres, siendo la excepción: Ingeniería Elec- trónica, Licenciatura en Filosofía y Lengua Castellana, Sociología, Ingeniería Mecánica y Estadística. Los resultados discriminados por Facultad y por género se encuentran en la tabla 3.

Tabla 3. Distribución del sedentarismo por género y por facultad.

\begin{tabular}{|c|c|c|c|c|}
\hline \multirow{2}{*}{ FACULTAD } & \multicolumn{2}{|c|}{ HOMBRES } & \multicolumn{2}{|c|}{ MUJERES } \\
\hline & TOTAL & SED & TOTAL & SED \\
\hline Ingeniería Ambiental & 42 & $22(52.4)$ & 49 & $34(69.4)$ \\
\hline Economía & 39 & $23(59.0)$ & 32 & $28(87.5)$ \\
\hline Comunicación Social & 44 & $24(54.5)$ & 66 & $44(66.7)$ \\
\hline Ingeniería Civil & 44 & $31(70.5)$ & 18 & $17(94.4)$ \\
\hline Negocios Internacionales & 66 & $41(62.1)$ & 111 & $90(81.1)$ \\
\hline Contaduría Pública & 26 & $19(73.1)$ & 41 & $34(82.9)$ \\
\hline Cultura física & 86 & $37(43.0)$ & 24 & $11(45.8)$ \\
\hline Ingeniería Electrónica & 28 & $16(57.1)$ & 3 & $1(33.3)$ \\
\hline Psicología & 8 & $6(75.0)$ & 50 & $40(80.0)$ \\
\hline Licenciatura en Filosofía y Lengua Castellana & 11 & $10(90.9)$ & 8 & $5(62.5)$ \\
\hline Derecho & 60 & $38(63.3)$ & 67 & $55(82.1)$ \\
\hline Ingeniería de Telecomunicaciones & 12 & $11(91.7)$ & 1 & $1(100.0)$ \\
\hline Administración de Empresas & 40 & $22(55.0)$ & 50 & $44(88.0)$ \\
\hline Sociología & 15 & $11(73.3)$ & 16 & $10(62.5)$ \\
\hline Ingeniería Mecánica & 37 & $24(64.9)$ & 4 & $2(50.0)$ \\
\hline Estadística & 11 & $8(72.7)$ & 7 & $5(71.4)$ \\
\hline No informado & 8 & $4(50.0)$ & 16 & $15(93.8)$ \\
\hline TOTAL & 577 & $347(60.1)$ & 563 & $436(77.4)$ \\
\hline Datos entre parénte & entarisn & al porcenta & & \\
\hline
\end{tabular}

Por último, la distribución por edad demostró que el 91.8\% de los estudiantes neotomasinos se encuentra entre los 16 y los 21 años. Una vez más, en cada uno de los grupos etarios, es mayor el sedentarismo de las mujeres comparado con el de los hombres, con una mayor tendencia a aumentarse en las mujeres, a medida que avanza la edad; en los hombres no se encuentra un patrón que relacione la edad con el sedentarismo (en Tabla 4 y Figura 2). 
Tabla 4. Distribución del sedentarismo por género y por rango de edad.

\begin{tabular}{|c|c|c|c|c|}
\hline \multirow{2}{*}{$\begin{array}{c}\text { RANGO DE EDAD } \\
\text { (Años) }\end{array}$} & \multicolumn{2}{|c|}{ HOMBRES } & \multicolumn{2}{c|}{ MUJERES } \\
\cline { 2 - 5 } & ACTIVOS & SED & ACTIVAS & SED \\
\hline$<16$ & 7 & $4(57.1)$ & 1 & $0(0.0)$ \\
\hline $16-18$ & 377 & $227(60.2)$ & 437 & $337(77.1)$ \\
\hline $19-21$ & 144 & $86(59.7)$ & 88 & $6877.3)$ \\
\hline $22-24$ & 32 & $21(65.6)$ & 22 & $17(77.3)$ \\
\hline$>25$ & 15 & $8(53.3)$ & 11 & $10(90.9)$ \\
\hline No informado & 2 & $1(50.0)$ & 4 & $4(100.0)$ \\
\hline TOTAL & 577 & $347(60.7)$ & 563 & $436(77.4)$ \\
\hline \multicolumn{4}{|c|}{ SED: Sedentarismo } \\
\hline
\end{tabular}

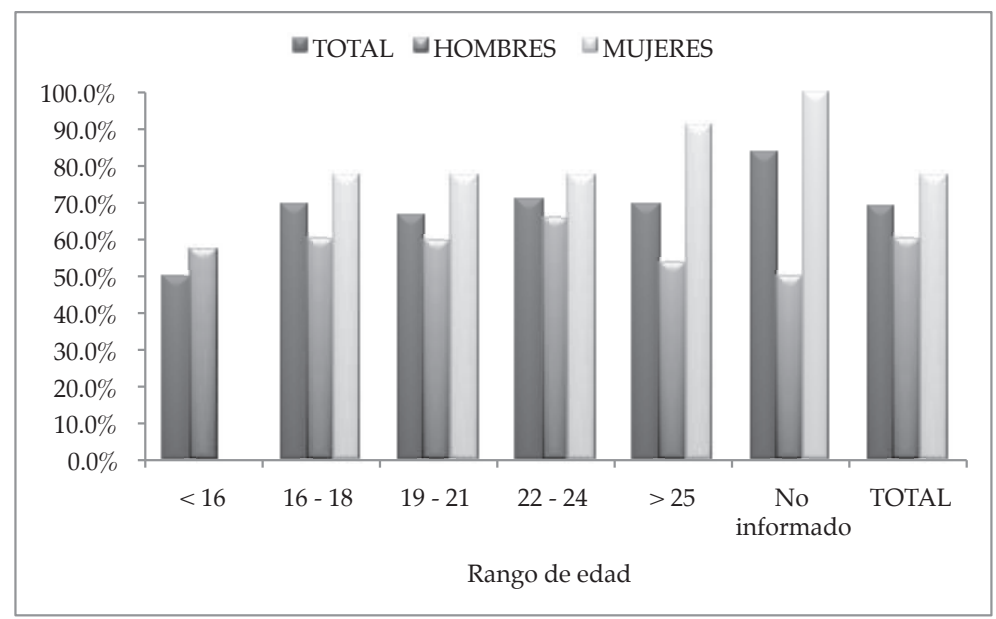

Figura 2. Representación gráfica de la distribución del sedentarismo por género y por rango de edad.

\section{Conclusión}

Varios estudios se han venido realizando en torno al tema del sedentarismo en la población universitaria. Un estudio argentino (Girotto, Vacchino, Spillmann \& Soria, 1996) presentó una prevalencia del sedentarismo del $51.9 \%$ en su población y un estudio chileno (Chiang, Casanueva, Cid, Gonzaléz, Olate, Nickel et al., 1999) arrojó un sedentarismo de $47.8 \%$ en hombres y $73.7 \%$ en mujeres.
En Colombia, un estudio de la Universidad Nacional dice que el sedentarismo en estudiantes de primer semestre es del $82.4 \%$ (Agencia de Noticias UN, 2009), mientras que la Universidad Jorge Tadeo Lozano publicó una cifra de sedentarismo del 88\% (Jerez, s. f.), esto demuestra que ya en varias ocasiones se ha tocado el tema del sedentarismo universitario. Sin embargo, la fortaleza de este estudio está en que por primera vez se aplica un test estandarizado para la cuantificación del sedentarismo, lo que permite que los resultados sean más fiables. 
Tratar de combatir el problema del sedentarismo en nuestros jóvenes nos obliga a intentar determinar su origen $y$, en este sentido, el resultado de varias investigaciones ha demostrado que el sedentarismo de los adultos es el resultado de hábitos sedentarios de la infancia, como la televisión o los videojuegos (Norman, Schmid, Sallis, Calfas y Patrick, 2005); prueba de esto son los datos publicados por el Instituto Colombiano de Bienestar Familiar (ICBF, 2006) que logró demostrar que, entre los 5 y 12 años, el $56.3 \%$ de los niños dedica 2 horas diarias o más a la televisión y los videojuegos y un $21.9 \%$ le dedica 4 horas o más. Por otro lado, las condiciones medioambientales y de infraestructura en las ciudades parecen hacer parte del problema, al limitar los espacios para la práctica deportiva (Coday, Klesges, Garrison, Johnson, Toole \& Morris, 2002).

\section{REFERENCIAS}

Agencia de Noticias UN. (2009, 2 de octubre). Sedentarismo afecta $80 \%$ de estudiantes en la UN. Recuperado del sitio Web de la Universidad Nacional de Colombia, http://www.agenciadenoticias.unal. edu.co/nc/detalle/article/ sedentarismoafecta-80-de-estudiantes-en-la-un/

Bassuk, S. S. \& Joann, E. M. (2005). Epidemiological evidence for the role of physical activity in reducing risk of type 2 diabetes and cardiovascular disease. Appl Physiol, (99), 1193-1204.

Bouchard, C., Tremblay, A., Leblanc, C., Lortie, G., Savard, R. \& Thériault, G. (1983). A method to assess energy expenditure in children and adults. Am J Clin Nutr, (37), 461-467.
Los estudiantes de primer semestre de la Universidad Santo Tomás deben sentirse orgullosos porque cuentan con un excelente espacio para la práctica del ejercicio físico, pero además porque durante el primer semestre gozan de la actividad física dirigida por profesionales, a través de la Cátedra de Formación Física Integral Henri Didón del Departamento de Humanidades y Formación Integral. Sin embargo, es necesario fortalecer los programas de actividad física y deporte encaminados a mantener, a lo largo de la vida universitaria, adecuados niveles de actividad física, que no solo aporten los beneficios ya antes nombrados, sino que además participen en la formación de hábitos de vida saludables que alejen a los jóvenes de problemas sociales como el alcohol y el tabaquismo.

Cabrera, A., Rodríguez, M. C., Rodríguez, L. M., Anía, B., Brito, B., Muros, M. et al. (2007). Sedentarismo: tiempo de ocio activo frente a porcentaje del gasto energético. Rev Esp Cardiol, 60 (3), 244250.

Chiang, M. T., Casanueva, V., Cid, X., González, U., Olate, P., Nickel, F. et al. (1999). Factores de riesgo cardiovascular en estudiantes universitarios chilenos. Salud Pública Mex, (41), 444-451.

Coday, M., Klesges, L. M., Garrison, R. J., Johnson, K. C., Toole, M. O, \& Morris, G. S. (2002). Health Opportunities with Physical Exercise (HOPE): Social Contextual Interventions to reduce Sedentary Behavior in Urban Settings. Health Educ Res, 17 (5), 637-647. 
Darren, E. R., Warburton, C. W. \& Shannon, S. D. (2006). Health benefits of physical activity: the evidence. CMAJ, 174 (6), 801-809.

Girotto, C. A., Vacchino, M. N., Spillmann, C. A. \& Soria, J. A. (1996). Prevalencia de factores de riesgo cardiovascular en ingresantes universitarios. Rev Saúde Pública, 30 (6), 576-586.

Instituto Colombiano de Bienestar Familiar, ICBF. (2006). Encuesta nacional de la situación nutricional en Colombia. Bogotá, Colombia: Imprenta Nacional de Colombia.

Jerez, A. C. (s. f.). Sedentarismo, cigarrillo y alcohol en universitarios. Recuperado del sitio Web de Colombia aprende, la red del conocimiento, http://www.colombiaaprende.edu.co/html/home/1592/article-81727.html
Mathieu, P., Poirier, P., Pibarot, P., Lemieux, I. \& Després, J. P. (2009). Visceral Obesity: The Link Among Inflammation, Hypertension, and Cardiovascular Disease. Hypertension, (53), 577-584.

Montoye, H. J., Kemper, H. C. G., Saris, W. H. M. \& Washburn, R. A. (1996). Measuring physical activity and energy expenditure. Champaign, IL, EE.UU.: Human Kinetics.

Norman, G. J., Schmid, B. A., Sallis, J. F., Calfas, K. J. \& Patrick, K. (2005). Psychosocial and Environmental Correlates of Adolescent Sedentary Behaviors. Pediatrics, (116), 908-916.

Thompson, P. D., Buchner, D. \& Piña, I. L. (2003). Exercise and Physical Activity in the Prevention and treatment of Atherosclerotic Cardiovascular Disease. Circulation, (107), 3109-3116. 